\title{
Bulk Gold/Bulk Rutile/Bulk Gold Sturdy Structure Resistive Gas Sensor for Exhaust Monitoring
}

\author{
Niloofar Dehghani, Ehsan Yousefiazari, Sepehr Aghili \\ Electronic Materials Laboratory, Electrical Engineering Department, K. N. Toosi University of \\ Technology, Tehran, 16317-14191, Iran \\ ndehghani@email.kntu.ac.ir
}

\begin{abstract}
:
A miniature, but sturdy, gas sensor capable of operation at temperatures as high as $600{ }^{\circ} \mathrm{C}$ is presented. The device comprises two back-to-back connected gold/rutile Schottky diodes, which are fabricated on the opposite bases of a self-standing pellet of polycrystalline rutile. The rutile layer is formed by the direct oxidation of titanium metal in air at $900^{\circ} \mathrm{C}$, and the Au/rutile contacts are formed by the diffusion bonding of the gold wire segments to the pellet bases. The current vs. voltage diagrams of the Au/rutile/Au structured device are recorded at different operating frequencies. The device current depends on the concentration of the oxygen vacancy at the Au/rutile interface and, hence, on the composition of the surrounding atmosphere. The current increases 15 times in response to the presence of 1000 ppm of ethanol vapor in air. Consisting only of bulk gold and bulk rutile, the device is resilient to harsh environments and elevated temperatures; a suitable gas sensor for in-exhaust installation.
\end{abstract}

Keywords: Gas sensor, oxygen vacancy, polycrystalline rutile, Schottky diode, memristor

\section{Introduction}

Metal-metal oxide-metal ( $\left.\mathrm{M}^{\prime} / \mathrm{MO} / \mathrm{M}^{\prime \prime}\right)$ structures have attracted considerable attention due to their advantagous in the operation of chemical gas sensors and memristors [1-3]. Here, we report fabricating robust $\mathrm{Au} / \mathrm{TiO}_{2} / \mathrm{Au}$ structures out of bulk rutile and bulk gold, recording their current-voltage (I-V) characteristics in different conditions, describing the anomalous asymmetry in the I-V diagrams based on the uneven distribution of the point defects in the rutile volume. The device shows its highest response in the $400-500{ }^{\circ} \mathrm{C}$ temperature range, which overlaps the temperature range experienced by a gas sensor in car engine exhaust.

\section{Methods}

$\mathrm{TiO}_{2}$ layers are grown on $10 \mathrm{~mm} \times 10 \mathrm{~mm}$ Ti chips cut from $1 \mathrm{~mm}$ thick titanium foil by thermal oxidation at $900{ }^{\circ} \mathrm{C}$ for 60 minutes in air. Samples are air quenched from the soaking temperature. After quenching, oxide layer can be separated easily from the surface of the Ti chip. The temperature vs. time profiles used for the oxidation and quenching are given in Fig. 1a. Owing to the heat capacity of the $\mathrm{Ti}$ substrate and the refractory underneath, the samples cool asymmetrically; the surface cools at a faster rate and, hence, a higher concentration of oxygen vacancy is "frozen" at and around the surface. Gold wire segments are formed as the top and bottom electrodes by diffusion bonding method [4]. A microheater made of a $10 \mu \mathrm{m}$ thick $\mathrm{SnO}_{2}$ deposited on alumina substrate, is attached to the underneath of the sample [5]. The schematic diagram of the device structure is shown in Fig. $1 \mathrm{~b}$.

\section{Results and discussion}

The I-V diagrams of the sample at five different voltage sweeping frequencies are shown in Fig. 2. At $0.01 \mathrm{~Hz}$, the device presents a rectifying diode-like I$\checkmark$ profile and a "soft breakdown" at around biasing voltage of $+3 \mathrm{~V}$. The observed resistance change is due to the motion of ionized oxygen vacancy on the surface of the grains, rather than the grain interior [2].

(a)

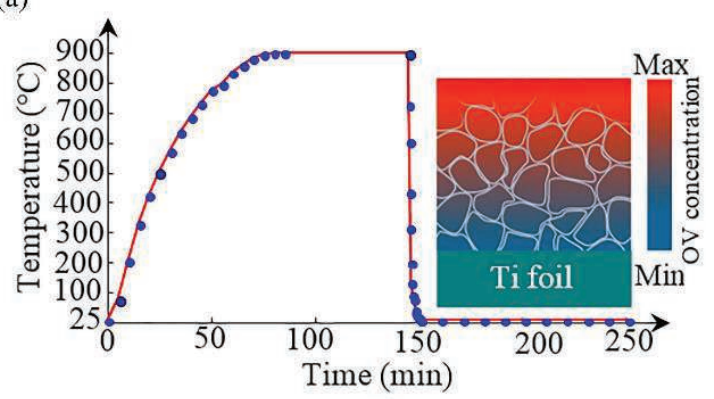

(b)

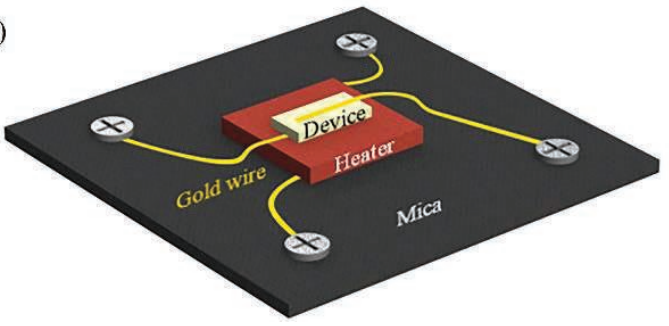

Fig. 1. The temperature vs. time diagrams of the annealing and quenching processes (a), and the schematic diagram of the device structure (b). 
The IOVs motion is in the direction of forming conductive filaments within the grain boundary regions. By increasing frequency, time available at each half cycle is not sufficient for IOVs to form substantial filaments. The implications of the proposed model are interesting as the surface conduction would allow influence of the external species on the conduction process. For instance, a high, fast, and reversible response to ethanol is expected as the ethanol molecules can readily interact with the IOVs available. This indicates the potential application of the device as a gas sensor.

The results for $1000 \mathrm{ppm}$ concentration of ethanol at $6 \mathrm{~V}$ biasing are summarized in Fig. 3, indicating that the useful operating temperature range of the device is wide spanning from $\sim 350{ }^{\circ} \mathrm{C}$ to $500{ }^{\circ} \mathrm{C}$. Upon exposure of the device to ethanol vapor, ethanol molecules decompose at the operating temperature. The produced radicals reduce the amount of surface adsorbed oxygen species and increase the surface concentration of the oxygen vacancies. Accordingly, the formation of the IOV filaments becomes more probable; current increase in constant conditions results in. The sensor response decreases for operating temperatures above $500{ }^{\circ} \mathrm{C}$, since at those temperatures, ethanol molecules tend to decompose before reaching the surface. Sensing mechanism of these devices have been attributed to both the chemoresistive properties of the gas sensitive oxide used and the fluctuations of the barrier height established at the $\mathrm{M}^{\prime} / \mathrm{MO}$ junction.

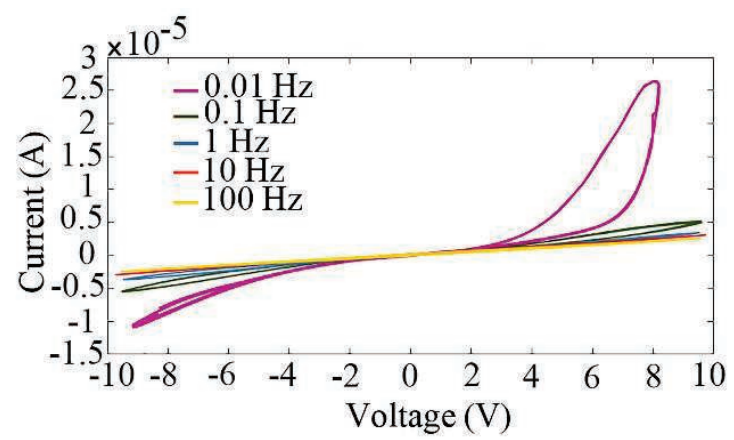

Fig. 2. The I-V characteristics recorded at the stated voltage sweeping frequencies.

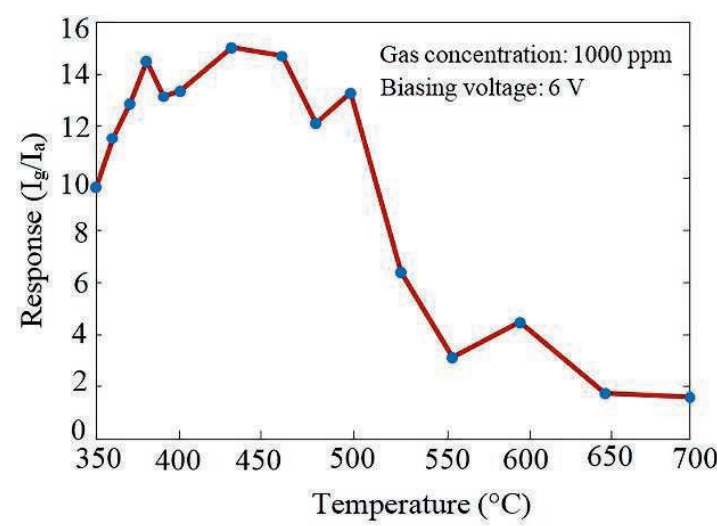

Fig. 3. The temperature dependence of the response magnitude of an $\mathrm{Au} / \mathrm{TiO}_{2} / \mathrm{Au}$ device to ethanol vapor at a constant concentration of 1000 ppm in air.

\section{Conclusions}

$\mathrm{Au} / \mathrm{TiO}_{2} / \mathrm{Au}$ devices present strongly asymmetric I-V characteristics at $0.01 \mathrm{~Hz}$. This anomaly was described based on the asymmetric distribution of the oxygen vacancy in the $\mathrm{TiO}_{2}$ volume. It was shown that the forward current of the asymmetric device is highly gas sensitive; it increases 15 times at the presence of $1000 \mathrm{ppm}$ ethanol vapor in air. The gas sensitivity of the device current was attributed to the increased OV concentration at the $\mathrm{TiO}_{2}$ grain surfaces, which facilitate IOV filament formation. The device is made of bulk rutile and bulk gold only, rendering the device sturdy and resilient at elevated temperatures as high as $520^{\circ} \mathrm{C}$ and harsh environs. The introduced device is suitable for in-exhaust operation.

\section{References}

[1] F. Hossein-Babaei and S. Rahbarpour, Separate assessment of chemoresistivity and Schottkytype gas sensitivity in $\mathrm{M}$-metal oxide- $\mathrm{M}^{\prime}$ structures, Sensors and Actuators B: Chemical, 160, 174-180, (2011); doi: 10.1016/j.snb.2011.07.029

[2] F. Hossein-Babaei and N. Alaei-Sheini, Electronic Conduction in Ti/Poly-TiO 2/Ti Structures, Scientific Reports, 6, 29624, (2016); doi: $10.1038 /$ srep29624

[3] M. Xiao, K. P. Musselman, W. W. Duley, and N. Y. Zhou, Resistive Switching Memory of TiO 2 Nanowire Networks Grown on Ti Foil by a Single Hydrothermal Method, Nano-Micro Letters, 9,15, (2017); doi: 10.1007/s40820-016-0116-2

[4] F. Hossein-Babaei, M. Gharesi, and M. Moalaghi, Diffusion Bonding of Metal Wires Directly to the Functional Metal Oxide Semiconductors for Forming Reliable Electrical Contacts, ACS applied materials \& interfaces, 9, 26637-26641, (2017); doi: 10.1021/acsami.7b07825

[5] F. Hossein-Babaei, M. Gharesi, and M. Ansari, Ten micron-thick undoped $\mathrm{SnO} 2$ layers grown by spray pyrolysis for microheater fabrication, Materials Letters, 196, 104-107, (2017); doi: 10.1016/j.matlet.2017.03.015 